\title{
RECENT RESULTS FROM RHIC SPIN AND BELLE FRAGMENTATION FUNCTION MEASUREMENTS
}

\author{
ANSELM VOSSEN \\ Center for Exploration of Energy and Matter, Indiana University \\ 2401 N. Milo B Sampson Lane, Bloomington, IN 47408, USA \\ avossen@indiana.edu
}

\begin{abstract}
This contribution summarizes recent results from the RHIC spin program and the measurement of light quark fragmentation functions at Belle.

Keywords: RHIC; PHENIX; STAR; spin; Belle; Collins Effect; interference fragmentation; fragmentation functions; transverse single spin asymmetries; gluon polarization.

PACS numbers: 13.85.Ni, 13.87.Fh,13.88+e
\end{abstract}

\section{Recent Results from RHIC Spin}

The Relativistic Heavy Ion Collider (RHIC) is located at Brookhaven National Laboratory. RHIC has the capability to collide polarized protons and a wide variety of ions at center of mass energies up to $500 \mathrm{GeV}$ for protons and up to $200 \mathrm{GeV}$ per nucleon for other ions. Transversely polarized protons are extracted from the polarized ion source, accelerated in the AGS ring and then injected into RHIC. There they are accelerated to the target energy and brought into collision at one of the interaction regions where the experiments are located. For longitudinal polarized protons, the spin is rotated from transverse polarization, which is stable in the ring, to longitudinal polarization by spin rotators which are located on both sides of the experiments. The two multipurpose detectors at RHIC are PHENIX $^{1}$ and STAR. ${ }^{2}$ Both detectors have complimentary capabilities. PHENIX was designed to detect rare probes. Its simultanous acceptance for charged hadrons and neutral pions covers only the detector pseudorapidity $\eta$ range $-0.35<\eta<0.35$ with two central arms that each have an angular coverage of about $90^{\circ}$. These arms are instrumented with electromagnetic calorimetry and drift/pad chambers to reconstruct charged tracks. The electromagnetic calorimeters have very fine granularity, allowing the reconstruction of neutral pions to high transverse momenta. Together with a high data taking rate, PHENIX is able to measure hadronic final states that decay into photons with very good precision. The other PHENIX detector system which is used in the spin measurements presented here is the Muon Piston Calorimeter (MPC) which is an electromagnetic calorimeter covering $3.1<\eta<3.9$ with full azimuthal coverage. It is used in the measurements of azimuthal asymmetries of $\pi^{0} \mathrm{~s}$ and $\eta$ 
mesons in forward directions. The STAR detector is built around a central time projection chamber (TPC) allowing precise tracking and particle identification by $\mathrm{dE} / \mathrm{dx}$ measurements with full azimuthal coverage in $-1.3<\eta<1.3$. The barrel electromagnetic calorimeter is built around the TPC, covering $-1<\eta<1$. Taken together with the endcap electromagnetic calorimeter $(1<\eta<2)$ and the forward meson spectrometer (FMS) $(2.5<\eta<4)$ STAR has nearly continuous coverage by electromagnetic calorimetry from $-1<\eta<4$. Both detectors have beam-beam counters and zero degree calorimetry which are used as local polarimeters. Before the FMS was installed in 2007, STAR used the Forward Pion Detector (FPD), which consisted of two modules of lead glass installed on either side of the beam pipe covering roughly the region $3.3<\eta<4$. The STAR detector with its larger acceptance, precise tracking but coarser EM-calorimetry and lower data taking rate is well suited for measuring jets and azimuthal correlations or charged hadrons.

\subsection{Cross-sections for Pions and Jets}

Both RHIC experiments have measured cross-sections for the final states used in the spin analysis. PHENIX published cross sections for $\pi^{0} \mathrm{~s}$ at $62.4 \mathrm{GeV}$ and $200 \mathrm{GeV}$ and STAR published cross-sections for jet and di-jet production at $200 \mathrm{GeV} .^{3-7}$ All cross sections show good agreement with NLO theory calculation, indicating that we are in a regime where perturbative QCD can be used reliably at least for spin-averaged observables and longitudinal double spin asymmetries above a $p_{T}$ of about $2 \mathrm{GeV} / \mathrm{c}$ where the cross-section follows a power law. It should be mentioned that the measurement at $62.4 \mathrm{GeV}$ could only be described properly after next-toleading-log resummation was used and the RHIC data provides an excellent test for this framework.

\subsection{Measurements of the Gluon and Sea Quark Polarization at RHIC}

One motivation for the spin physics program at RHIC was to access the gluon helicity distribution. At mid-rapidity, the underlying partonic scattering processes are gluon-gluon and quark-gluon. Only at high $p_{T}$ a significant contribution of quarkquark scattering exists (about $40 \%$ at $p_{T}=8 \mathrm{GeV} / \mathrm{c}$ ). The longitudinal double spin asymmetry $A_{L L}$ is defined as $A_{L L}=\frac{\sigma^{++}-\sigma^{+-}}{\sigma^{++}+\sigma^{+-}}$, where $\sigma^{++}$and $\sigma^{+-}$are the differential production cross-sections of pions or jets when the helicities of the colliding protons are parallel or anti-parallel. As previously described, the flagship result sensitive to the gluon polarization at PHENIX is $A_{L L}$ from $\pi^{0}$ mesons, whereas STAR uses jets and di-jets. ${ }^{8} 9$ The advantage of the latter is that the analysis of jet measurements is independent of the choice of fragmentation functions and jets, in particular di-jets, are more sensitive to the kinematics of the scattered partons. Therefore the $x$ of the polarized gluon distribution that is probed can be determined more precisely than it could be in single hadron production. On the other hand, the measurement of $A_{L L}$ for neutral pions at PHENIX can take advantage of high 
data taking rates and very well understood calorimeters that have very good energy resolution and granularity. This results in highly precise measurements for $A_{L L}$ in particular at small and moderate particle $p_{T}$. STAR uses the midpoint cone jet algorithm to reconstruct jets in the detector. Figure 1 shows the results from 2006 and 2009 STAR data compared with theory curves from the DSSV and GRSV groups. Both, DSSV and GRSV are fits of PDFs to world data. GRSV ${ }^{10}$ fits polarized DIS data and is included mainly to point out the difference between the assumption of zero gluon polarization (GRSV-ZERO) and a non-vanishing gluon polarization (GRSV-STD). The current DSSV takes all recent world data up to RHIC run 6 into account and provides estimates of uncertainties. With these uncertainties, the extracted polarized gluon distribution is not significantly different from zero and leads to the prediction ${ }^{11}$ that is shown in Fig. 1. The run 9 STAR jet and di-jet results, which are not yet included in this fit, both show that they have the statistical power to reduce the error bars on current fits significantly. In fact there is indication, that the new STAR results are evidence for a non-zero gluon polarization in the region probed by the experiment. ${ }^{12}$ In comparison, PHENIX $A_{L L}$ measurements also shown in Fig. 1, do not show a significant non-zero value ${ }^{14}$ yet, even though there seems to be an indication of an excess over the theory curve. However, due to the difference in phase space probed, the experiments are still consistent.

Another missing part of our understanding of the spin structure of the proton is the helicity distribution of sea quarks. Here the parity violating single-spin asymmetry $A_{L}$ is measured, which is related to the $\mathrm{u}$ and d (anti-)quark helicity distributions by

$$
A_{L}^{W^{+}}=\frac{-\Delta u\left(x_{a}\right) \bar{d}\left(x_{b}\right)+\Delta \bar{d}\left(x_{a}\right) u\left(x_{b}\right)}{u\left(x_{a}\right) \bar{d}\left(x_{b}\right)+\bar{d}\left(x_{a}\right) u\left(x_{b}\right)} .
$$

A similar relation holds for $A_{L}^{W^{-}}$. Using asymmetric collisions one can select events in which $x_{a}<<x_{b}$ and vice versa. Thereby one can cleanly access $\Delta \bar{u}$ and $\Delta \bar{u}$. To access this region, both PHENIX and STAR implemented detector upgrades
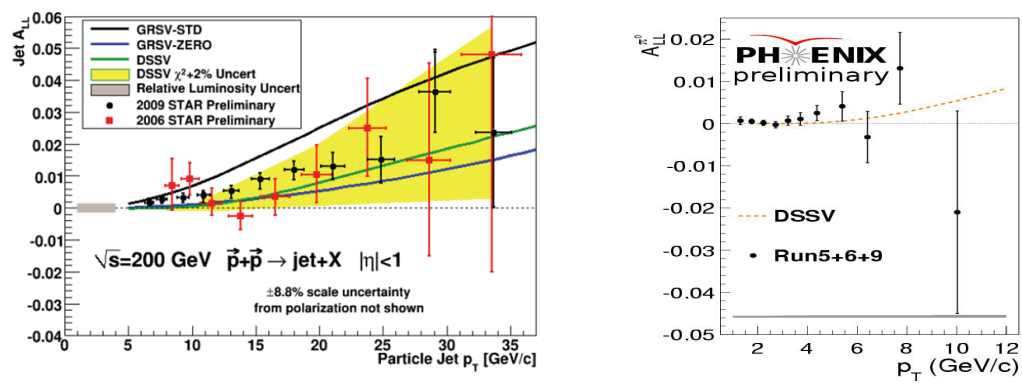

Fig. 1. Longitudinal double spin asymmetry $A_{L L}$ from jets at STAR and neutral pions at PHENIX. See text for description. 
that allow them to detect the lepton from the $W$ decay in a kinematic region corresponding to detector- $\eta$ of about $1<\eta<2$. So far, only exploratory results for $A_{L}$ and the $W$-production cross-sections have been published ${ }^{15,16,17}$ but analysis of the recently taken run 12 dataset with much larger statistics and higher polarization is under way. RHIC expects to collect more data at $\sqrt{s}=500 \mathrm{GeV}$ in the coming years to improve on these measurements as well.

\subsection{Measurement of transverse single spin asymmetries at RHIC}

With our improved understanding of the mechanism behind transverse single spin asymmetries in SIDIS and $\mathrm{p}+\mathrm{p}$ it was realized that these effects can shed light on novel aspects of the partonic structure of the nucleon, like the transversity distribution of quarks and correlations between spin and transverse momentum in nucleon structure and fragmentation. ${ }^{18}$ The connection of intrinsic transverse momentum and orbital angular momentum make these measurements particularly interesting. In the following, I will review recent results for the transverse single spin asymmetry (TSSA) $A_{N}$ and asymmetries in correlations, such as the Collins effect and di-hadron asymmetries. The latter allow to select specific mechanisms that lead to the asymmetries.

\subsubsection{Measurement of the TSSA $A_{N}$}

The transverse single spin asymmetry of single pions $A_{N}$ is a left-right asymmetry of the produced particle yield and is usually defined as the amplitude of the modulation of the normalized difference between spin-up and spin-down cross-sections in the azimuthal angle $\Phi$ between polarization vector and hadron momentum corrected for the polarization $P$ :

$$
\frac{1}{P} \frac{\sigma^{\uparrow}-\sigma^{\downarrow}}{\sigma^{\uparrow}+\sigma^{\downarrow}}=A_{N} \sin (\Phi) .
$$

PHENIX and STAR measured $A_{N}$ for both neutral pion and eta mesons in the forward direction with the FPD/FMS and the MPC, respectively. In this kinematic region the effect has been observed over a wide range of $\sqrt{s}$ from $19.4 \mathrm{GeV}$ up to $500 \mathrm{GeV} \cdot{ }^{18,19,20}$ All data so far on $\pi^{0} A_{N}$ indicate scaling in Feynman $x$, $x_{F}=p_{L} / p_{L}^{\max }$. Large asymmetries are observed at large $x_{F}$, At $x_{F}=0.6$ asymmetries of about $10 \%$ are reached. Around $x_{F}=0$ and negative $x_{F}$ no significant asymmetries are observed. Since at LO in 2-2 parton scattering $x_{F}$ is related to the $\mathrm{X}$-values of the partons scattering off each other, $x_{F}=x_{1}-x_{2}$, large $x_{F}$ are sensitive to valence quark properties, whereas small $x_{F}$ probe the sea. Since we know that most transverse spin effects are stronger in the valence region, we can understand the enhancement of the forward asymmetries. New results by STAR have indicated that the asymmetry of $\eta$ 's might be larger than the asymmetries of $\pi^{0}$ 's, ${ }^{21}$ a property which is not yet fully understood and might point to large spin dependencies of the strange quark distribution in the proton. ${ }^{22}$ The other recent experimental 

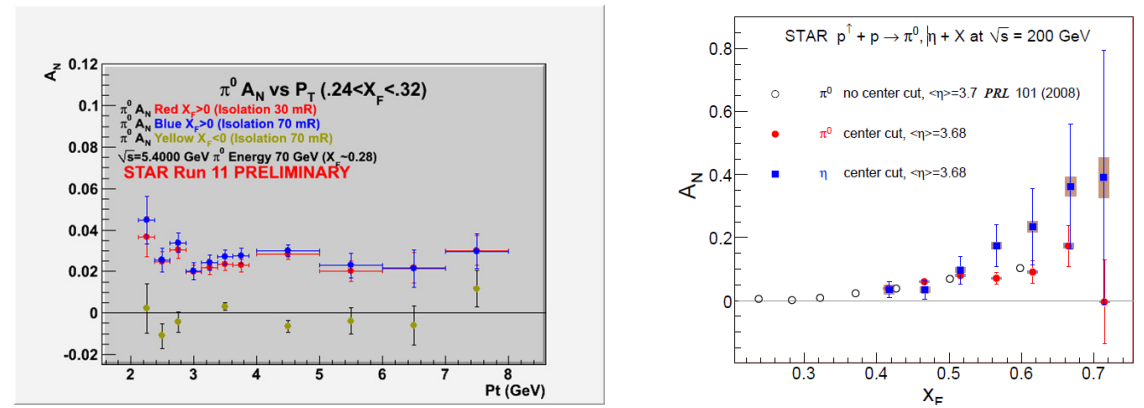

Fig. 2. Transverse single spin asymmetry $A_{N}$ for $\pi^{0} \mathrm{~s}$ measured at STAR at $\sqrt{s}=500 \mathrm{GeV}$ and for $\pi^{0} \mathrm{~s}$ and $\eta \mathrm{s}$ at $\sqrt{s}=200 \mathrm{GeV}$ at forward rapidities. Even at $p_{T}$ greater than $4 \mathrm{GeV} / \mathrm{c}$, signifcant asymmetries are observed. Asymmetries for $\eta$ mesons are slightly larger than $A_{N}$ for $\pi^{0}$.

advances are measurements at high $p_{T}$ in 200 and $500 \mathrm{GeV}$ collisions. Results are shown in Fig. 2. At these transverse momenta it is expected that intrinsic transverse momentum does not play a role anymore and the asymmetries should be describable in the collinear twist-3 framework. ${ }^{23,24}$ However, a fundamental prediction of this framework is a $1 / p_{T}$ dependence of the asymmetries which has not been observed yet. New PHENIX and STAR data show a flattening of the asymmetries, but there is no significant fall yet. There are no firm predictions where the turnaround is expected to happen, however a common scale of intrinsic $p_{T}$ is about $1 \mathrm{GeV} / \mathrm{c}$, so the experimental data is including this region.

\subsubsection{Correlation Measurements}

The mechanism behind the transverse spin asymmetry $A_{N}$ is still unknown. If factorization holds, there are two mechanisms that were proposed. Either transversely polarized quarks fragment via the Collins fragmentation function for which the momentum of the produced hadron is correlated with the fragmenting quark spin or the parton distribution function correlates intrinsic quark transverse momentum with the nucleon spin. At high transverse momenta the transverse momentum can be generated by the hard scattering by coupling to an additional parton from the nucleon or the fragmentation function. In the first case, one has to introduce twist-3 distribution functions and in the latter case twist- 3 fragmentation functions. Since the transverse momentum now originates in the hard part, a collinear framework can be used for the soft parts. In the kinematic overlap region both approaches, transverse momentum dependent (TMD) and twist-3 distributions are equivalent. This has been proven for the Drell-Yan process. ${ }^{25}$ To isolate effects of TMD PDFs one can measure asymmetries of jets where fragmentation effects are integrated over. STAR has measured $A_{N}$ for jets in the mid-rapidity region. The asymmetries are not significantly different from zero, hinting at a vanishing Sivers effect for gluons. ${ }^{26}$ The transverse polarization of quarks in hadrons is described by the 
transversity distribution function. This function is the least well known of the three leading twist distribution functions that are needed to describe the spin structure of the nucleon at leading twist. It is chiral-odd and can only be measured if coupled to another chiral-odd function. This is usually a fragmentation function which correlates the transverse polarization of the fragmenting quark with the transverse momenta of the final state hadrons and that leads to correlations between the final state hadrons and the jet-axis. STAR and PHENIX are measuring di-hadron correlations by extracting an asymmetry similar to Eq. (2) but $\Phi$ is the azimuthal angle between the difference vector between the two hadrons $\vec{R}=\vec{P}_{h_{1}}-\vec{P}_{h_{2}}$ and the proton polarization vector with respect to the vector sum of the two hadron momenta. Using the fragmentation function extracted at Belle as explained in the next section, transversity can be extracted from these measurements. STAR can also measure azimuthal correlations in jets, like the Collins effect, that are sensitive to transversity and this measurement is underway.

\section{Belle}

The Belle experiment at KEK stopped data taking in 2009. Before that, it collected over $1 a b^{-1}$ of integrated luminosity of $e^{+} e^{-}$collisions mainly at the $\Upsilon(4 S)$ resonance at $10.58 \mathrm{GeV}$. But Belle took about $10 \%$ of the data off-resonance where mostly light and charm quarks are produced. Belle has excellent pion id and good kaon id (about $85 \%$ purity at $10 \%$ pion fake rate) over a wide momentum range using $\mathrm{dE} / \mathrm{dx}$, aerogel cherenkov counters and time of flight detectors. Recently Belle extracted the first yields for identified pion and kaons from the off-resonance data set. $^{27}$ These results are shown in Fig. 3. From these yields, the unpolarized fragmentation functions of pions and kaons can be extracted. The precision is several orders of magnitude better than what has been previously available mainly from LEP data. The center of mass energy of Belle is also closer to the SIDIS and polarized $\mathrm{p}+\mathrm{p}$ experiments. Together with the precise data at high $z$, this makes also a much improved extraction of the gluon fragmentation function possible. The Belle data will be included in global fits, such as $\operatorname{DSS}^{28}$ to extract fragmentation functions which are a necessary ingredient to all semi-inclusive measurements with single hadrons in the final state. In addition to the unpolarized yields, spin dependent fragmentation functions are measured from the full dataset. By using the thrust of the event to select two-jet events, the fraction of events containing B-mesons is reduced to less than $2 \%$. Since Belle is using unpolarized beams, the spin dependent fragmentation functions have to be extracted from correlations of azimuthal asymmetries between two back-to-back jets using the fact, that the produced quark-antiquark pair has aligned spins. If the jets are approximately perpendicular to the beam axis, the quark polarization with respect to the jet axis is transversal. The Collins angle can then be defined as the azimuthal angle of the hadron with the event plane around the jet axis. Similarly for the di-hadron interference fragmentation function, the relevant angle is between $\vec{R}$ and the event event plane. These angles $\Phi$ are 


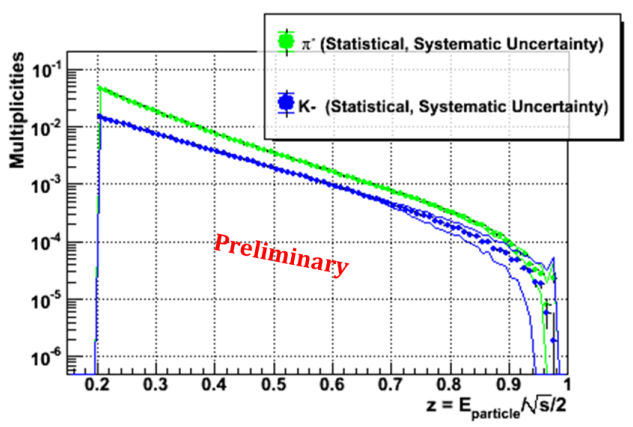

Fig. 3. Identified negative pion and kaon multiplicities measured at Belle. See text for description.

measured for each hadron (pair) and all combinations between the two sides. The measured yield has then a dependence on $\cos \left(\Phi_{1}+\Phi_{2}\right)$, where $\Phi_{1}$ is measured in one hemisphere and $\Phi_{2}$ in the other. The amplitude of this modulation is then directly proportional to the product of Collins (IFF) fragmentation functions of the quark and antiquark. Since gluon radiation leads to additional azimuthal asymmetries which are not canceled in the measurement of single hadrons, the final observable for the Collins effect are modulations of the ratio between different charge combinations. Here the gluon contribution cancels, since the gluon coupling is charge independent, however the Collins effect is charge dependent, and therefore survives. Recent results for IFF can be found in Ref. [29]. The Collins FF has been extracted in Ref. [30]. Collins results from Belle have been used in the first global analysis of transversity and the IFF results have been used for the first point-by-point extraction of transversity from SIDIS data in a collinear framework. ${ }^{31}$

\section{Outlook}

In the near future the spin program at RHIC will concentrate on the $\mathrm{W}$ program. Both experiments implemented forward upgrades to detect leptons from $\mathrm{W}$ decay up to a detector- $\eta$ of 2 . PHENIX and STAR developed decadal plans ${ }^{32}$ which call for upgrades for spin physics in the more forward region up to $\eta=4$ with capabilities for jet reconstruction and particle ID. One of the main goals for this upgrade is to measure transverse spin dependent observables in the valence region, where effects are large and where they have not been measured by SIDIS experiments at a high scale. In $\mathrm{p}+\mathrm{p}$ one can in particular investigate questions about TMD evolution, universality, the role of gauge links and the origin of the transverse spin asymmetry $A_{N}$. At Belle we are currently analyzing data to extract Collins fragmentation functions for charged kaons, neutral pions and $\eta$ 's as well as vector mesons. Future plans include the measurement of jet-handedness and azimuthal correlations in dihadron pair production that are sensitive to the longitudinal IFF $G_{1}^{\perp}$. Observing these effects in $e^{+}+e^{-}$would help our understanding of the TMD framework we use in SIDIS, since it gives well defined predictions for this process. Currently 
we are also preparing for the start of Belle $\mathrm{II}^{33}$ which is expected to see beam in 2014. This successor of the Belle experiment will have improved particle ID and vertexing capabilities in addition to sampling a luminosity which is about a factor 40 higher than before. The added statistics and improved detector will enable the extraction of statistics hungry observables like $p_{T}$ dependent fragmentation functions. Fragmentation functions with kaons in the final state will be improved as well as the charm tagging for light quark FF measurements.

\section{References}

1. W. A. Zajc et al. [PHENIX Collaboration], Nucl. Phys. A 698, 39 (2002).

2. K. H. Ackermann et al. [STAR Collaboration], Nucl. Instrum. Meth. A 499, 624 (2003).

3. A. Adare et al. [PHENIX Collaboration], Phys. Rev. D 76, 051106 (2007).

4. A. Adare et al. [PHENIX Collaboration], arXiv:1209.3283 [nucl-ex].

5. A. Adare et al. [PHENIX Collaboration], arXiv:1202.4020 [hep-ex].

6. A. Adare et al. [PHENIX Collaboration], Phys. Rev. D 79, 012003 (2009).

7. M. Ploskon [STAR Collaboration], Nucl. Phys. A 830, 255C (2009).

8. P. Djawotho [STAR Collaboration], arXiv:1106.5769 [nucl-ex].

9. T. Sakuma et al. [STAR Collaboration], J. Phys. Conf. Ser. 295, 012068 (2011).

10. M. Gluck, E. Reya, M. Stratmann and W. Vogelsang, Phys. Rev. D 63, 094005 (2001).

11. D. de Florian, R. Sassot, M. Stratmann and W. Vogelsang, Phys. Rev. D 80, 034030 (2009).

12. D. de Florian, R. Sassot, M. Stratmann and W. Vogelsang, Prog. Part. Nucl. Phys. 67, 251 (2012).

13. A. Adare et al. [PHENIX Collaboration], Phys. Rev. Lett. 106, 062001 (2011).

14. A. Adare et al. [PHENIX Collaboration], Phys. Rev. Lett. 103, 012003 (2009).

15. M. M. Aggarwal et al. [STAR Collaboration], Phys. Rev. Lett. 106, 062002 (2011).

16. A. Adare et al. [PHENIX Collaboration], Phys. Rev. D 83, 032001 (2011).

17. L. Adamczyk et al. [STAR Collaboration], Phys. Rev. D 85, 092010 (2012).

18. A. Vossen [STAR Collaboration] J. Phys. Conf. Ser. 316, 012004 (2011) and references therein.

19. S. Heppelmann [STAR Collaboration] Talk at CIPANP 2012, to be published.

20. J. Koster, Ph.D. Thesis, University of Illinois at Urbana-Champaign (2010).

21. L. Adamczyk et al. [STAR Collaboration], Phys. Rev. D 86, 051101 (2012).

22. K. Kanazawa and Y. Koike, Phys. Rev. D 83, 114024 (2011).

23. A. V. Efremov and O. V. Teryaev, Phys. Lett. B 150, 383 (1985).

24. J. -w. Qiu and G. F. Sterman, Phys. Rev. Lett. 67, 2264 (1991).

25. X. Ji, J. -W. Qiu, W. Vogelsang and F. Yuan, Phys. Rev. Lett. 97, 082002 (2006).

26. L. Adamczyk et al. [STAR Collaboration], Phys. Rev. D 86, 032006 (2012).

27. M. Leitgab [Belle Collaboration], Talk at DIS 2012, to be published.

28. D. de Florian, R. Sassot and M. Stratmann, Phys. Rev. D 76, 074033 (2007).

29. A. Vossen et al. [Belle Collaboration], Phys. Rev. Lett. 107072004 (2011).

30. R. Seidl et al. [Belle Collaboration], Phys. Rev. D 78, 032011 (2008).

31. A. Bacchetta, A. Courtoy and M. Radici, Phys. Rev. Lett. 107, 012001 (2011).

32. STAR and PHENIX Decadal Plans, available from http://www.bnl.gov/npp .

33. T. Abe [Belle II Collaboration], arXiv:1011.0352 [physics.ins-det]. 